\title{
Translation Process of Caused-Motion Constructions from the Blending Point of View
}

\author{
Zeng-qiang XIA \\ School of Foreign Languages, Henan Institute of Engineering, Zhengzhou, China \\ 450000
}

52453091@163.com

Keywords: Translation process, Caued-motion construction, Point of view.

\begin{abstract}
Conceptual integration (blending) provides a way of the mental processes of mappings between languages and thoughts. If a source text is treated as the blends of the source language structures and schemata of the communicative events, in translating, when translators digest the source text, translators are actually disintegrating the text, and disclose the schemata of the communicative events integrated by the ST, and when actually translating, translators integrate the digested schemata from the source text with the target language structures.
\end{abstract}

\section{Introduction}

In this section, the researcher discusses some issues pertaining to translation studies in the frame of blending. First of all, the researcher puts forward conceptual blending point of view towards translation, which suggests that the analysis of the ST means de-integrate the schematic structures, then, the translator integrates the schematic structures with corresponding syntactic patterns in the TT. Translation process is a bi-lateral integration of the ST and TT, both syntactically and schematically. According to the relations between the source and target languages in terms of communicative mode, translation processes can be classified into four types of integration networks: simplex and mirror networks, as well as single-scope and double-scope networks.

\section{Translation is the Result of Bilateral Integration of the ST and TT}

The perspective of blending, translation is the result of the bi-literal integration of the ST and TT. Translator receives the linguistic expressions of the ST as an input, which triggers the conceptual structures of the ST, then; the communicative mode in a culture in turn takes shape, by which schemata of the communicative event can be drawn. Up to this point, the first stage of translation is accomplished, which can be considered as the reverse process of blending.

As to any kind of ST, it must be the schemata of the ST that take shape first, then, through the integration of the communicative mode in a culture and the semiotic system which concretely behaves all kinds of genres such as novel and poetry in macro level and concrete wording and sentencing in micro level, the TT finally can take shape. Simply speaking, a text is an integration of a certain kind of communicative mode in a culture with a certain kind of semiotic system. As to the first stage of translation, the de-generation is a reverse of the blending, as have mentioned before, with the chief mission of the translator at this stage can be considered as disclosing subjective imagination structures of the ST. In fact, de-integration is a rather opening process, which means different translators may obtain different schemata of the communicative events based on different experiential 
backgrounds of their own. Having obtained the schemata of the communicative events, the translator enters into the second stage of translation, during which translator aims at seeking the effective communicative mode in a culture. It then, the translator integrates the mode with the semiotic system in the target language, thus the TT is accomplished. Up to this point, the whole process of translation is completed.

Whereas, the re-integration is far from easy in the process of translation practice. It is how to choose the communicative mode in a culture in the target language that annoys translators most. Firstly, take the culture of the target language itself as consideration, translators have different expressive modes with one event; secondly, there always exist many choices of the communicative modes in the cultures between the source and target languages. Therefore this choice will be an everlasting opening process, which can give an explanation why a ST has so many different TTs. The relations between the source and target languages in terms of communicative mode can be:

A. Totally the same, which means that the imaginative objects and the thus constructed image schemata in both source and target languages have the same pragmatic function in the process of translation;

B. Partially the same, which means that two different imaginative objections in the source and target languages have the same pragmatic function in the process of translation;

C. Totally different, which means that the same imaginative objection in the source and target languages has totally different pragmatic functions in the process of translation;

D. Lacking, which means that one imaginative objection in the communication mode in a culture does not exist in another. Serious considerations are worth taking on how to adjust the lacking, which is also an opening process.

\section{Four Types of Integration Networks}

According to the blending theory, human cognitive activities are governed by a series of rules, which based on four types of integration networks: simplex networks, mirror networks, single-scope networks and double-scope networks. (Fauconnier and Turner, 2004:119-135). Since translation can be considered as a kind of communication, translation processes also be classified into four types of integration. In the next section, four types of networks of translation will be introduced one by one.

Simplex networks and mirror networks belong to the mental mapping that is shaped in a same constructive frame. That is, all the four spaces INPUT1, INPUT2, the generic space and the blending have the same framing structure. Simplex and mirror integration networks reflect the common cognitive structures of human beings, which cannot be disturbed by different communicative modes in different cultures. In the translation process, it means that the imaginative objects in the communicative mode and thus constructed schemata of the communicated events, together with their pragmatic functions are totally the same between the source and the target languages.

In the thesis, the translation of CM constructions belongs to this category. As will be argued in the next chapter, both English and Chinese share a common conceptual structure to express CM constructions, that is, A causes B move to C, which expresses a simple event that takes place all around the world. Therefore, in the process of translation, cultural factors can be neglected and attention should be transferred to grammatical blending between the ST and TT. Single-scope networks contain the blending that INPUT1 and INPUT2 have different constructive frames, which share 
some similarities and each can be interpreted with the other. The frame of the blending only comes from one of the input space, INPUT1 or INPUT2, which is selected by the translator. In the process of de-integration, the translator may reconstruct the schemata of the author with his own experiences as the constructive frames, thus the translator can disintegrate and reconstruct the ST just by his own understanding, or translator may refurbish his own encyclopedia knowledge with the author's schemata. As have mentioned above, due to the differences of translators' encyclopedia knowledge and the different styles of the reconstruction and refurbishment, the selective process is an everlasting changing and opening one. In the process of translation, the selection is between domestication and foreignization. If schemata of the communicative events of the source language are selected as the frames of the blending, the selection should be considered as foreignization; if schemata of the communicative events of the target language are selected as the frame of the blending, the selection should be considered as domestication. The translator can make a choice by the purpose of translation, which is a subjective choice by the agent. Generally speaking, it is the schemata of the communicative events and the thus exerting pragmatic functions between the source and target languages that trigger the emergence of single-scope networks in the process of translation.

Double-scope networks have most complex integration modes, of which the constructive frames of the blends come from both INPUT1 and INPUT2, both of which have rather complex constructive frames. For instance, INPUT1 and INPUT2 may share no analogical characteristics or even have upside-down causalities, while it is in this blending process that new ideas, conceptual structures and communicative modes are created. Therefore, it is still a selective problem on how and how much does the constructive frame of the blending come from that of INPUT1 and INPUT2. In the process of de-integration, the translator may face the problem that his experiences cannot map with that of the author's. Sometimes the translator in vain tries to refurbish the constructive frames of the ST, therefore he has to abandon both frames and create new ones. This kind of de-integration is creative in nature, for the schemata that created by the translator are new to both himself and the author of the ST. So, it is obviously that the created ones are neither created by foreignization nor domestication; they are totally new integrations. As single-scope and double-scope networks are irrelevant to the study of the thesis, discussion in detail will be omitted here.

Conceptual integration (blending) provides a way of the mental processes of mappings between languages and thoughts. If a source text is treated as the blends of the source language structures and schemata of the communicative events, in translating, when translators digest the source text, translators are actually disintegrating the text, and disclose the schemata of the communicative events integrated by the ST, and when actually translating, translators integrate the digested schemata from the source text with the target language structures. Translating is, as a matter of fact, mental processes of embodied minds; a way to illustrate how the same communicative event is mapped onto different language structures in different translators' mind. Cognitive models of conceptual integration offer penetrating insights in this regard.

In the frame of conceptual integration, the relations between source and target languages in terms of the communicative mode can be: totally the same; partially the same; totally different and lacking. Corresponding to the four relations translation processes can be classified as four types of integration networks: simplex networks, mirror networks, single-scope and double-scope networks. As to E-C translation of 
the CM constructions, translators should focus their attention on the simplex and mirror networks since both English and Chinese share the same communicative mode to express $\mathrm{CM}$ concept with no cultural divergences. In the following section, an elaborate study on CM constructions in English and Chinese in the frame of blending should be carried out, so as to lay a foundation for the contrastive and translation studies in the late chapters. In the first part of next chapter, introduction of a brief literature review on the studies of CM constructions is necessary.

\section{Conclusion}

From the mapping analysis in Chapter IV, Fauconnier and Turner suggest analyzing the examples as cases of blending between prototypical instances of the CM constructions, (as in Jack threw the ball into the basket) and a novel conceived caused-motion sequences of events. One input structure is the causal sequence of the conceived events in the world. The second input structure is a schematic representation of a prototypical $\mathrm{CM}$ event. The blending between these input structures are based on conceived structural similarities. The blending resulting is an extension of the basic (prototypical) use of the constructions. That is to say, the blending analysis emphasizes how CM constructions are shaped in English in a static perspective that is irrelevant to communication, which is far from enough since the study on $\mathrm{CM}$ constructions in the thesis aims at laying a foundation for the translation process of $\mathrm{CM}$ constructions. Thus, $\mathrm{CM}$ constructions should be viewed in an environment of communication, that is, from the point of construction generation and interpretation. Blending analysis performed between a prototypical instance of the $\mathrm{CM}$ construction and a conceived CM event sequence is far from enough; the analysis should performed additionally between an abstract representation of the $\mathrm{CM}$ constructions and the conceived CM events. That is to say, in the additional analysis, input 2 is not a representation of any actual sentence in the language, but rather a representation of the construction's form and semantic-conceptual schema that is abstracted from all instances of the construction. Thus far, CM constructions are being put in connection with semantic approach and translation process can be seen more clearly in the late section.

\section{References:}

[1] Nida, E. (2001). Language and Culture. Shanghai: Shanghai Foreign Language and Education Press.

[2] Nida, E. (1969). Science of Translation. In Anwar S. Dil, Language and Thought. Essays by Eugene A. Nida. Stanford: Stanford University Press.

[3] Nida, E. (1964). Towards a Science of Translation. Leiden: Brill.

[4] Oakley, T. (1995). Presence: the conceptual basis of rhetorical effect. Ph.D. dissertation, University of Maryland.

[5] Turner, M. (1992). Design for a Theory of Meaning. The Nature and Ontogenesis of Meaning, Lawrence Erlbaum Associations, pp. 91-107. 